\title{
Tagung der Sektion Pathophysiologie und Aerosolmedizin in der DGP
}

\author{
Anne Hilgendorff \\ G. Hüls \\ L. Gortner \\ H. Lindemann
}

Berlin, 21. - 22. November 2003

\section{Erratum}

Erratum zur Tagung der Sektion Pathophysiologie und Aerosolmedizin in der DGP, Berlin, 21.-22. November 2003. Pneumologie 2003; 57: $763-770$

Bei der Publikation der Abstracts zur Herbsttagung der Sektion wurde der Beitrag von Frau Dr. Hilgendorff versehentlich nicht gedruckt.
Stellenwert der multiplen Okklusionstechnik zur Bestimmung der Lungendehnbarkeit im Rahmen der Lungenfunktionsdiagnostik bei Säuglingen mit bronchopulmonaler Dysplasie.

Anne Hilgendorff, G. Hüls, L. Gortner, H. Lindemann

Zentrum für Kinderheilkunde und Jugendmedizin, Universitätsklinik Gießen, Deutschland

Die bronchopulmonale Dysplasie (BPD) bleibt eine wichtige Komplikation im Langzeitverlauf als Folge eines Atemnotsyndroms bei Frühgeborenen. Neben definierten klinischen und radiologischen Kriterien, die zur Diagnosestellung herangezogen werden, fehlen jedoch hinreichend aussagekräftige Variablen zur Differenzierung der pulmonalen Veränderung. Zu ihrer Etablierung wurden frühgeborene Säuglinge(FG) mit der Diagnose einer BPD, definiert als Sauerstoffbedarf am 28. Lebenstag, prospektiv einer Lungenfunktionsdiagnostik zugeführt. Zum errechneten Geburtstermin konnten 8 FG mit der Diagnose einer BPD im Master Screen Baby Body (Fa. Jaeger, Hoechberg, Deutschland) bodyplethysmografisch untersucht werden. Es wurden Atemwegswiderstand und funktionelle Residualkapazität ( $\mathrm{FRC}_{\text {box. }}$ ) ermittelt. Zur näheren Charakterisierung der pulmonalen Schädigung erfolgte zusätzlich die Messung der Lungencompliance bezogen auf die Atemruhelage $\left(\mathrm{C}_{\mathrm{L}} / \mathrm{FRC}_{\mathrm{box}}\right)$ mit Hilfe der multiplen Okklusionstechnik (MOT). Die Messungen erforderten eine milde Sedierung der Patienten. Trotz eines immer unauffälligen Auskultationsbefundes zeigte sich bei vier FG ein mittelgradig bis deutlich erhöhter exspiratorischer Atemwegswiderstand. Eine Überblähung fand sich bei drei Patienten. Bei vier FG ließ sich eine deutliche Erniedrigung des $\mathrm{FRC}_{\text {box }}$ nachweisen in Zusammenhang mit fehlenden bis nur milden Veränderungen im Sinne einer obstruktiven Ventilationsstörung. Sowohl die $C_{L} / F C_{\text {box }}$ als auch das Vorliegen obstruktiver Ventilationsstörungen oder Bestehen eines Volumenmangels zeigte eine tendentielle Abhängigkeit vom Gestationsalter. Fazit: Zur Einschätzung des Anteils obstruktiver und restriktiver Ventilationsstörungen am klinischen Bild der BPD erscheint die differenzierte Lungenfunktionsdiagnostik, für die die MOT eine wichtige Ergänzung und Erweiterung darstellt, gut geeignet. 